$\xi=-1$

\title{
Model-based compressed sensing algorithms for MIMO- OFDM channel estimation
}

\author{
P. N. Jayanthi ${ }^{1 *}$, S Ravishankar $^{2}$ \\ ${ }^{1}$ Research scholar, Dept. of ECE, $R$ V College of Engineering, Bengaluru, India \\ ${ }^{2}$ Professor, Dept. of ECE, $R$ V College of Engineering, Bengaluru, India \\ *Corresponding author E-mail:
}

\begin{abstract}
High data rates on the wireless channel can be achieved by combining orthogonal frequency division multiplexing (OFDM) with multiple input multiple output (MIMO) communication modulation scheme. MIMO-OFDM system impulse response of the channel is approximately sparse. Sparse channelestimation can be done using Compressive Sensing (CS) techniques. In this paper, a low complexity model based CoSaMp Compressive Sensing (CS) algorithm with conventional tools namely Least Square (LS) and Least Mean Square (LMS) are used for MIMO-OFDM channel estimation. Simulation results show amodel based CoSaMP for MIMO-OFDM channel estimation with LMS tool the Normalized Mean Square Error(NMSE) reduced by $34 \%$ with very reduced complexity.
\end{abstract}

Keywords: Sparsity; Compressedsensing; Channelestimation; Compressed Sampling Matching Pursuit; Model-Based Cosamp.

\section{Introduction}

MIMO-OFDM is the air-interface solution for thepresent 5G wireless communication system. Using MIMO- OFDM system diversity and multiplexing of the wireless channel can be exploited. Wireless channels are frequency selective in nature. This frequency selective fading of a multipath can be reduced using OFDM technique [1].Most of the wireless standards employ pilot based channel estimation technique to decode the transmitted data bits. Pilot symbols are to be transmitted on subcarriers which for the initial channel estimation which reduces the spectral efficiency. But Wireless communication channels tend to exhibit therich multipath channels, which are sparse in nature [2], [3].The conventionalmethods used do not utilize the channel sparsity of the wireless channel. Estimation of these kinds of sparse channel and Compressive Sensing (CS) algorithms are used with few pilots compared to the conventional methods [4] for OFDM system. CS algorithms like OMP and SP are used for OFDM and MIMO OFDM channelestimation using LS tool [5]-[6].An adaptive channel estimation technique namely LMS is used for the channel estimation MIMO-OFDM using different CS algorithms. Computational complexity of SP, OMP and CoSaMP is $\mathrm{O}(\mathrm{m} . \mathrm{N} . \log (\mathrm{K})$ ), $\mathrm{O}(\mathrm{K} . \mathrm{m} . \mathrm{N})$ and $\mathrm{O}(\mathrm{m} . \mathrm{N})$ respectively [7]. Here $\mathrm{m}$ and $\mathrm{N}$ is a number of rows and columns in the measurement matrix respectively and $\mathrm{K}$ is the sparsity in the measurement matrix.

All the conventional CS algorithms considered,assume the positions of the sparse signal. However, actual signals include some structure in the information. This structure can be effectively used to reconstructthe original signal in a better way [8]. The model based CoSaMp CS algorithm is proposed for the MIMO-OFDM channel estimation in this paper. Additional rules are added for the reconstruction process for the model-based CS algorithms. By adding some extra rules the reconstruction of the signals can be improved. Particularly, assuming these sparse signals have some extra fundamental structure. In addition, the model-based
CoSaMP is used for the channel estimation which uses the Restricted Amplification Property [RAP] instead of Restricted Isometric Property [RIP] to decrease the robustness of the CoSaMP [9].

In this work, our focus is to use model-based CoSaMP with LS and LMS tools for MIMO-OFDM channel estimation, which are sparse in nature. The performances of conventional CoSaMp and model-based CoSaMp for MIMO-OFDM channel estimation are compared. MIMO-OFDM system model is described in Section II. Conventional CoSaMP and Model-based CoSaMP algorithms are illustrated in section III. Complexity comparision is shown in section V. In section VI NMSE vs SNR for these algorithms are compared with each other. The model-based CoSaMp outperforms to other CoSaMP algorithms with less computational complexity and BER and Section VII is concluded with these results and the references are listed in section VIII.

\section{MIMO-OFDM system}

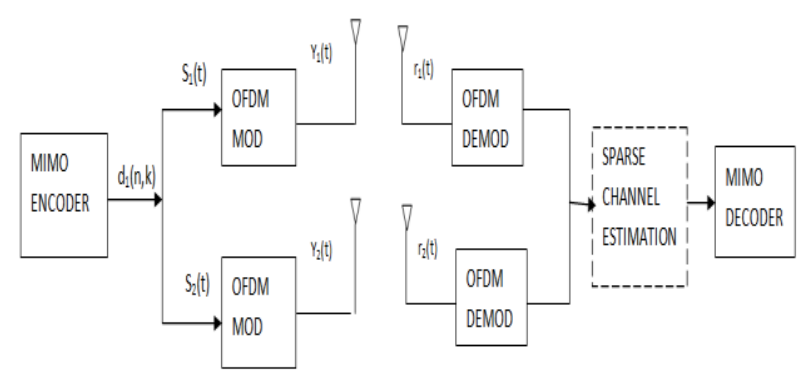

Fig. 1: MIMO- OFDM Structure.

Figure 1 shows the basic system for the MIMO-OFDM considered for this work.Due to the reflection, refraction and scattering properties of the electromagnetic wave in the wireless communication, the Channel Impulse Response [CIR] generally contains a few 
dominant paths hence the CIRs are sparse nature. The encoded data are modulated using OFDM and transmitted using $2 \times 2$ MIMO. At the receiver, these received data are demodulated using OFDM. The CIR is estimated using the pilot's symbols and CIRs in MIMO -OFDM systems are given by

$$
H_{y}=F_{N M_{r}}\left[\begin{array}{c}
h_{y} \\
0_{(N-L) M_{r} \times M_{t}}
\end{array}\right]
$$

Where

$$
\mathrm{H}_{\mathrm{y}}=\left[\begin{array}{c}
\mathrm{H}_{0, \mathrm{y}} \\
\vdots \\
\mathrm{H}_{\mathrm{N}-1, \mathrm{y}}
\end{array}\right] ; \mathrm{F}_{\mathrm{NM}_{\mathrm{r}}}=\mathrm{F}_{\mathrm{N}} \otimes \mathrm{I}_{\mathrm{M}_{\mathrm{r}}}
$$

Since FFT size $\mathrm{N}$ is more than channel length $\mathrm{L}$, from FFT matrix $\mathrm{F}_{\mathrm{NM}_{\mathrm{r}}}$ only the first LMrcolumns are involved in the calculation. The equation (1) can also be written as

$$
\mathrm{Hy}=\mathrm{F}_{\mathrm{N}} \mathrm{Mr}(1: \mathrm{LMr}) \text { hy }
$$

To reduce the effect of noise augmentation, the pilot subcarriers are equally placed. The channel frequency response at these pilot positions is shown below.

$$
\mathrm{H}_{\mathrm{y}}^{(\mathrm{P})}=\left[\begin{array}{c}
\mathrm{H}_{\mathrm{P}, \mathrm{y}} \\
\vdots \\
\mathrm{H}_{\mathrm{P}+(\mathrm{L}-1) \mathrm{M}, \mathrm{y}}
\end{array}\right] ; \mathrm{W}_{\mathrm{N}}^{(\mathrm{P})}=\left[\begin{array}{lll}
1 & & \\
& \mathrm{~W}_{\mathrm{N}}^{\mathrm{p}} & \\
& & \mathrm{W}_{\mathrm{N}}^{\mathrm{P}(\mathrm{L}-1)}
\end{array}\right] \otimes \mathrm{INr}
$$

Where $W_{N}=e^{-\frac{j 2 \pi}{N}}$ is a Fourier matrix. $P$ is any integer such that $0 \leq \mathrm{b} \leq \mathrm{y}-1 . \mathrm{H}_{\mathrm{y}}^{(\mathrm{P})}$ is the $\mathrm{P}$-the downsampled version of the $\mathrm{H}_{\mathrm{y}}$ and $\mathrm{W}_{\mathrm{N}}^{(\mathrm{P})}$ is used for shift operation of order $\mathrm{P}$.

$$
\mathrm{p}=0,1 \ldots \mathrm{M}-1
$$

$$
\mathrm{H}_{\mathrm{y}}^{(\mathrm{P})}=\mathrm{F}_{\mathrm{LM}_{\mathrm{r}}} \mathrm{W}_{\mathrm{N}}^{(\mathrm{P})} \mathrm{hy}
$$

Where $\mathrm{F}_{\mathrm{LM}_{\mathrm{r}}}$ is an $\mathrm{LM}_{\mathrm{r}} \times \mathrm{LMr}$ DFT matrix. From the downsampled variant of Hy, i.e., $\mathrm{H}_{\mathrm{y}}^{(\mathrm{P})}$, the time domain CIR hy can be obtained. Eqn. (3) shows that the CIR can be obtained through pilot tone set.

\section{Compressed sensing}

\section{a) Compressed Sensing}

When the signal considered as sparse or compressible in nature CS is capable of fully recovering the original signal. Instead of sampling at the Nyquist rate, it takes into consideration fewer measurements than traditional sampling theory demands [10]-[12] With suitable basis functions CS can recover many signals with only a few number of sparse coefficients. From equation (4) Q sparse vector $h \in \mathbb{R}^{\mathrm{L}}$ can be recovered by considering $\mathrm{Ad} \in \mathbb{R}^{\mathrm{Np} \times \mathrm{L}}$ by resolving $1_{0}$ - norm minimization difficulty [13]

$\min h \in \mathbb{R}^{\mathrm{L}}\|\mathrm{h}\|_{0}$ s.t. $\left\|\mathrm{y}-\mathrm{A}_{\mathrm{d}} \cdot \mathrm{h}\right\|_{2} \leq \sigma_{\mathrm{n}}$

Here $\|\mathrm{h}\|_{0}$ istotal number sparse coefficients of $\mathrm{h}$ and $\sigma_{\mathrm{n}}$ variance of noise. The solution for this is NP hard and integrative. However, these kinds of problems can be solved using convex optimization algorithms or greedy algorithms [11].

$\min \mathrm{h} \in \mathbb{R}^{\mathrm{L}}\|\mathrm{h}\|_{1}$ s.t. $\left\|\mathrm{y}-\mathrm{A}_{\mathrm{d}} \cdot \mathrm{h}\right\|_{2} \leq \sigma_{\mathrm{n}}$

Nevertheless, convex optimization algorithms are complex and these algorithms are not appropriate for the time-varying channel. Greedy algorithms are used for time-varying channel because frequent channel estimation is required. b) Compressed sensing for MIMO OFDM channel estimation Consider MIMO-OFDM system with number of multipath is equal to $\mathrm{L}$. The CIR of this system is given by [14]

$\mathrm{h}(\tau, \mathrm{t})=\sum_{\mathrm{q}=1}^{\mathrm{L}} \alpha_{\mathrm{q}}(\mathrm{t}) \delta\left(\tau-\tau_{\mathrm{q}}(\mathrm{t})\right)$

Where $\tau_{\mathrm{q}}(\mathrm{t}) \in \mathbb{R}$ and $\alpha_{\mathrm{q}}(\mathrm{t}) \in \mathbb{C}$ delay spread and magnitude for path q. For block fading channel with steady parameters and neglecting its synchronization symbol, equation (6) can be written in discrete form with sampling interval $\mathrm{T}_{\mathrm{q}}$

$\mathrm{h}(\tau, \mathrm{t})=\sum_{\mathrm{q}=1}^{\mathrm{L}} \alpha_{\mathrm{q}}(\mathrm{t}) \delta\left(\left(\tau-\tau_{\mathrm{q}}\right) \mathrm{T}_{\mathrm{q}}\right)$

Assuming total number channel taps are $\mathrm{L}$ and $\mathrm{K}$ of themnonzero $(\mathrm{K}<<\mathrm{L})$ and it is called as $\mathrm{K}$-sparse channel. Let us consider OFDM system with $\mathrm{N}$ subcarriers. Out of $\mathrm{N}$ subcarriers select $\mathrm{Np}$ subcarriers as pilots with position $\mathrm{t} 1, \mathrm{t} 2, \ldots \ldots \mathrm{tNp} \quad(1 \leq$ $\mathrm{t} 1<\mathrm{t} 2<\ldots .<\mathrm{tNp} \leq \mathrm{N})$ and remaining subcarriers are used as data carriers. Let us consider locations, $\mathrm{X}(\mathrm{t} 1), \mathrm{X}(\mathrm{t} 2), \ldots \ldots, \mathrm{X}(\mathrm{tNp})$ and $\mathrm{Y}(\mathrm{t} 1), \mathrm{Y}(\mathrm{t} 2), \ldots \ldots . \mathrm{Y}(\mathrm{tNp})$ are the symbols transmitted and $\mathrm{t}$ received respectively for the pilot symbols. For these pilot positions, the estimatedfrequency domain transfer functionis estimated by

$$
\widehat{\mathrm{H}}(\mathrm{k})=\frac{\mathrm{Y}(\mathrm{k})}{\mathrm{X}(\mathrm{k})} \mathrm{k}=\mathrm{t} 1, \mathrm{t} 2 \ldots . \mathrm{tNp}
$$

To use of channel sparsity, it can be formulated as

$$
\mathrm{y}=\mathrm{X} \cdot \mathrm{F}_{\mathrm{NpXL}} \cdot \mathrm{h}+\mathrm{n}
$$

Here $\quad \mathrm{X}=\operatorname{diag} \quad\{\mathrm{X}(\mathrm{t} 1), \mathrm{X}(\mathrm{t} 2), \ldots \ldots, \mathrm{X}(\mathrm{tNp})\}, \mathrm{h}=[\mathrm{h}(1)$ $\ldots ., \mathrm{h}(\mathrm{L})]^{\mathrm{T}}$ and $\mathrm{n}_{0}=\left[\mathrm{n}_{0}(1), \mathrm{n}_{0}(2), \ldots \ldots \mathrm{n}_{0}(\mathrm{~Np})\right]^{\mathrm{T}}$ are the diagonal matrix,the CIR and the AWGN noise vector respectively. $\mathrm{Y}=[\mathrm{Y}(\mathrm{t} 1), \mathrm{Y}(\mathrm{t} 2) \ldots \mathrm{Y}(\mathrm{tNp})]$ and

$\mathrm{F}_{\mathrm{N}_{\mathrm{p}} \times \mathrm{L}}=\frac{1}{\sqrt{\mathrm{N}}}\left[\begin{array}{cccc}1 & \mathrm{w}^{\mathrm{t}_{1}} & \cdots & \mathrm{w}^{\mathrm{t}_{1}(\mathrm{~L}-1)} \\ 1 & \mathrm{w}^{\mathrm{t}_{2}} & \cdots & \mathrm{w}^{\mathrm{t}_{2}(\mathrm{~L}-1)} \\ \vdots & \vdots & \ddots & \vdots \\ 1 & \mathrm{w}^{\mathrm{t}_{\mathrm{N}_{p}}} & \cdots & \mathrm{w}^{\mathrm{t}_{\mathrm{N}}(\mathrm{L}-1)}\end{array}\right]$

Here $\mathrm{w}=\mathrm{e}^{-\mathrm{j} 2 \pi / \mathrm{N}}$. In fact $\mathrm{F}_{\mathrm{N}_{\mathrm{p}} \times \mathrm{L}}$ is a DFT sub matrix preferred by column indices $[0,1 \ldots \mathrm{L}-1]$ and row indices $\left[\mathrm{t}_{1}, \mathrm{t}_{2}, \ldots \ldots \mathrm{t}_{\mathrm{Np}}\right]$ from a regular NxN Fourier matrix[14].

$\mathrm{y}=\mathrm{A}_{\mathrm{c}} \cdot \mathrm{h}+\mathrm{n}_{0}$

Where $\mathrm{A}_{\mathrm{c}}=\mathrm{X} . \mathrm{F}_{\mathrm{N}_{\mathrm{p}} \times \mathrm{L}}$

To estimate the channel $\mathrm{h}$ should be obtained from $\mathrm{y}$ and $\mathrm{A}_{\mathrm{c}}$.If columns of $A_{c}$ is less than its rows, equation (5) can be solved with LS problem[4]

$\hat{\mathrm{h}}_{\mathrm{ls}}=\left(\mathrm{A}_{\mathrm{d}}^{\mathrm{H}} \mathrm{A}_{\mathrm{d}}\right)-1 \mathrm{~A}_{\mathrm{d}}^{\mathrm{H}} \cdot \mathrm{y}$

MIMO OFDM channel estimation can be done by estimating SISO OFDM simultaneously. Frequency orthogonal pilots are used for individual transmitters with sparse recovery algorithms.

\section{Reconstruction algorithms}

Originally, lo minimization technique wasused to find the correct solution to CS problems. In the 10"norm" finds its number of nonzero entries. Because of employing CS, one canincrease the net data transmissionrate, since only a fraction of the samples transmitted compared to a number of samples in aconventional sampling and compression encoder. This technique also reduces the size of thememory unit required to store the data samples. Compressive sensing is also a stable process, which means that even if the data samples are corrupted by noise, the system can still recon- 
structa very good quality approximation of the signal, given that the system quantizes the signalreasonably fine without the reconstruction process going unstable. The signal is randomly sampled andtransmitted across to the receiver. The randomly sensing matrix will be shared with the receiver.This sensing matrix is essential to reconstruct the signal back. Any third-party interceptors who try to intercept the signal will not be able to fully reconstruct the signal back, as they are not having knowledge about the sensing matrix.In conventional CS algorithms, RIP is asufficient condition for the steady recovery of both sparse and compressiblesignalmeasurements through an iterative, greedy search. Algorithms such as MP, OMP,SP[15]-[16] andCoSaMP [10], all revolvearound the bestapproximation for the estimated signal.In SP, instead of selecting one column at each step as in OMP, S columns are selected from the measurement matrix iteratively until stopping criteria is met.Therefore, the computational time complexity of is reduced in SP than OMP.

a) $\mathrm{CoSaMP}$

An extension to OMP algorithm is the CoSaMP,which has tighter bounds on its convergence and performance. The algorithm used for CoSaMp is shown in algorithm1,whichconsists of five main steps,

b) Identification: It identifies the major2scoefficients of the signal.

c) Support Merge: Merges the support of the signal proxy with the support of the solutionfrom the previous iteration.

d) Estimation: Finds a solution using least squares.

e) Pruning: After finding the solution estimate and it compresses to the required support.

f) Sample Update: The "sample" is updated.

\begin{tabular}{|c|c|}
\hline & Algorithm 1: CoSaMP \\
\hline \multicolumn{2}{|l|}{ Input: } \\
\hline & $\mathrm{S}$ is sparsity \\
\hline - & $Y \in R m$ and the measurement matrix $\Phi \in R m x n$ \\
\hline - & Procedure: \\
\hline - & $\mathrm{x} \in \mathrm{R}^{\mathrm{n}}$ such that $\hat{\mathrm{x}}$ is sparse and $\mathrm{y}=\Phi \hat{\mathrm{x}}$ \\
\hline & $\mathrm{x}(0) \leftarrow(0)$ \\
\hline & $\mathrm{v} \leftarrow \mathrm{y}$ \\
\hline & $\mathrm{k} \leftarrow 0$ \\
\hline iv) & till the condition is false perform \\
\hline & $\mathrm{k} \leftarrow \mathrm{k}+1$ \\
\hline & $\mathrm{z} \leftarrow \Phi T v$-Sparse proxy \\
\hline vii) & $\Omega \leftarrow \operatorname{supp}(\mathrm{x}(\mathrm{k}-1))$-Support model based residual estimation \\
\hline viii) & $\Gamma \leftarrow \Omega \cup \operatorname{supp}(\mathrm{x}(\mathrm{k}-1))-$-combine supports \\
\hline & $\hat{\mathrm{x}} \leftarrow \operatorname{argmin} \hat{\mathrm{x}}: \operatorname{supp}(\hat{\mathrm{x}})=\Gamma\|\Phi \hat{\mathrm{x}}-\mathrm{y}\| 2$-Least square solution \\
\hline & $x(k)=\hat{x} s$-Prune w.r.t signal model \\
\hline xi) & $\mathrm{v} \leftarrow \mathrm{y}-\Phi \mathrm{x}(\mathrm{k})$ - current sample is updated. \\
\hline xii) & end while \\
\hline xiii) & $\hat{\mathrm{x}} \leftarrow \mathrm{x}(\mathrm{k})$ \\
\hline xiv) & Return $\hat{x}$ \\
\hline
\end{tabular}

The computational complexity is $\mathrm{O}(\mathrm{m}$. $\mathrm{N})$. which is less compared to other conventional CS algorithms.

g) Model-basedcs algorithms

A model-based reconstruction algorithms are used for the compressible signal which exploits thestructural property of the signal. In model-based RAmP is used, which is the equivalent to the RIP used in the conventional CS. In these algorithms, wavelet trees and block sparsity are integrated into CS recovery algorithms and hence they provide recovery from just $\mathrm{M}=\mathrm{O}(K)$ measurements. Model-based CS theory provides a set of rules to generate structured signal recovery algorithms with good performance.Structured sparsity models provide advantages to CS. It reduces the total measurements required to extract a signal. During signal recovery, they can discriminate actual signal from the estimated signal so that more robust recovery of the signal is possible.

h) Model-based CoSaMP
CoSaMPis chosen for model-based because it provides robust recovery almost equal to the best convex optimization based algorithms. In addition, it hasa simple iterative, greedy structure for sparse estimation.The CoSaMP algorithm tomodel-basedCS is comparatively easy. Fundamentally, it involves two changes to theCoSaMP algorithm in steps vii and $\mathrm{x}$. As an alternative to taking the best $\mathrm{s}$ and $2 \mathrm{~s}$ sparse estimation, just take the best model based approximations at these steps, which can be seen in modelbasedCS algorithm.

\begin{tabular}{|c|c|}
\hline \multirow{2}{*}{\multicolumn{2}{|c|}{ Input: }} \\
\hline & \\
\hline & $\begin{array}{l}\text { - } \mathrm{S} \text { is sparsity } \\
\text { - } \mathrm{Y} \in \mathrm{Rm} \text { and the measurement matrix } \Phi \in \mathrm{Rmxn} \\
\text { - } \mathrm{A} \text { model Ms } \\
\text { - } \quad \mathrm{X} \in \mathrm{R}^{\mathrm{n}} \text { such that } \mathrm{x} \text { is sparse and } \mathrm{y}=\Phi \hat{\mathrm{x}}\end{array}$ \\
\hline i) & $\mathrm{x}^{(0) \leftarrow(0)}$ \\
\hline ii) & $\mathrm{v} \leftarrow \mathrm{y}$ \\
\hline iii) & $\mathrm{k} \leftarrow 0$ \\
\hline iv) & till the condition is false perform \\
\hline v) & $\mathrm{k} \leftarrow \mathrm{k}+1$ \\
\hline vi) & -Sparse proxy \\
\hline $\begin{array}{l}\text { vii) } \\
\text { viii) }\end{array}$ & $\begin{array}{l}\Omega \leftarrow \operatorname{supp}\left(\mathrm{M}_{2}(\mathrm{z}, \mathrm{s})-\text { combine model based residual estimation }\right. \\
\Gamma \leftarrow \Omega \cup \operatorname{supp}\left(\mathrm{x}^{(\mathrm{k}-1)} \quad \text {-merge supports }\right.\end{array}$ \\
\hline ix) & $\hat{\mathrm{x}} \leftarrow \operatorname{argmin} \hat{\mathrm{x}}: \operatorname{supp}(\hat{\mathrm{x}})=\Gamma\|\Phi \hat{\mathrm{x}}-\mathrm{y}\|_{2}$-Least square solution \\
\hline $\mathrm{x})$ & $\mathrm{x}^{(\mathrm{k})}=\widehat{\mathrm{X}}^{\mathrm{s}} \quad$-Prune wrt signal model \\
\hline xi) & $\mathrm{v} \leftarrow \mathrm{y}-\Phi_{\mathrm{x}}(\mathrm{k})$-Update current sample. \\
\hline xii) & end while \\
\hline xiii) & $\hat{\mathrm{X}} \leftarrow \mathrm{x}^{(\mathrm{k})}$ \\
\hline xiv) & Return $\hat{x}$ \\
\hline
\end{tabular}

Figure 2 shows the relative performance for model-based and conventional based CoSaMP, from [12]. By looking at thereconstructionusing the model based CoSaMP is better.

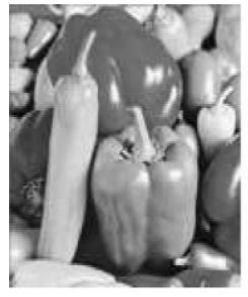

(a)

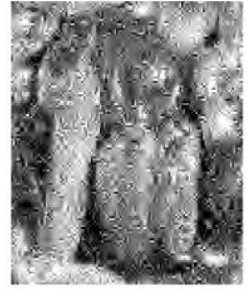

(b)

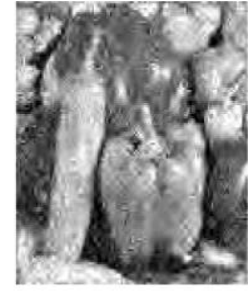

(c)
Fig. 2: Illustration Performance of Model Based and Conventional Cosamp Reconstruction. (A) The Original Image with $\mathrm{N}=128 \mathrm{X} 128=16$, 384 Pixels and the Images Reconstruction Using $M=5000$ Measurements (B) Image Recovered from Conventional Cosamp Algorithm (C) the Model Based Cosamp Recovery [12].

\section{Comparison of complexity}

Complexity analysis of theconventional CS reconstruction algorithms and the model based CoSaMP are tabulated in Table1.

Table 1: Complexity Analysis of Cs Algorithms

\begin{tabular}{ll}
\hline Total Tx. antennas & 2 \\
\hline Total of Rx. antennas & 2 \\
Channel model & Rayleigh \\
Number of Input Samples & $10^{-7}$ \\
Number of subcarriers & $\mathrm{N}=256$ \\
Number of pilot subcarriers & $\mathrm{Np}=12$ \\
Number of cyclic prefix & $\mathrm{N}_{\mathrm{G}}=64$ \\
Delay spread & 15 \\
Doppler frequency & $0.1 \mathrm{~Hz}$ \\
Type of Modulation & $\mathrm{QAM}$ \\
\hline
\end{tabular}




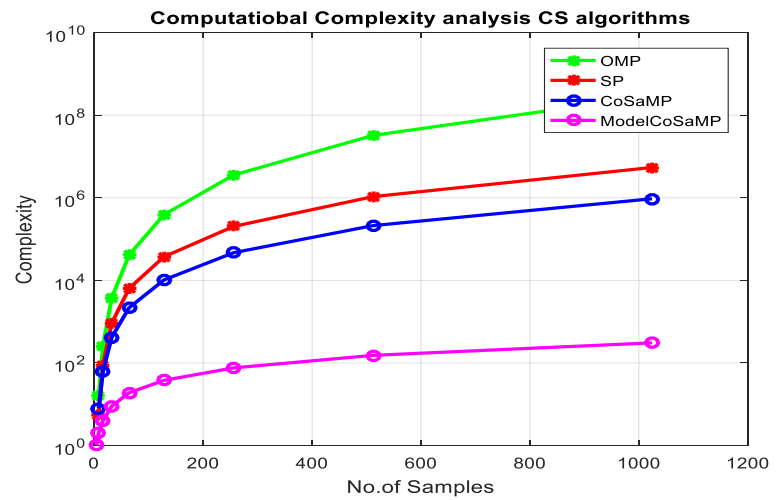

Fig. 3: The Plot of Computational Complexity Analysis of Conventional Andmodel-Based CS Algorithms.

Fig. 3 shows the computational complexity study for the conventional and the model-based CS algorithms. In model-based CoSaMP wavelet trees and block sparsity are integrated into CS recovery algorithms and hence they providerobust recovery from just $\mathrm{O}(\mathrm{K})$ measurements.

\section{Simulation results}

In this paper, MIMO OFDM channel is estimated using the conventional CS like OMP, SP and CoSaMP and model-based CoSaMP algorithms combined with LS and LMS tools. Total numbers of subcarriers used are 256. Out of 256 subcarriers, just 12 are used as pilot subcarriers. The parameters considered for the MIMO-OFDM system are tabulated in Table 2.

Table 2: Parameters Considered for Simulation

\begin{tabular}{ll}
\hline CS Reconstruction algorithms & Complexity \\
\hline OMP & O(K. m. N) \\
SP & O(m. N. log (K)) \\
CoSaMP & O (m. N) \\
Model-based CoSaMP & O (K) \\
\hline
\end{tabular}

Figure 4 NMSE vs SNR (dB) for conventional CS with amodel based CoSaMP for MIMO-OFDM channel estimation with LS tool.

Fig. 4 gives the performance comparisonof conventional and the model based CoSaMP compression technique with LStool forMIMO-OFDMsystem channel estimation. All CS algorithms consider the channel sparsity for estimation and the reconstruction will be better than LS tool alone. And also the among conventional CS algorithms CoSaMp performs better since it iteratively selects $2 \mathrm{~K}$ atoms using amatched filter. Performance of model-based $\mathrm{CoSaMP}$ is $34 \%$ better than conventional CoSaMP since it imposes the modelcompressiblesignal and the interrelatedRAmP condition on the measurement matrix.

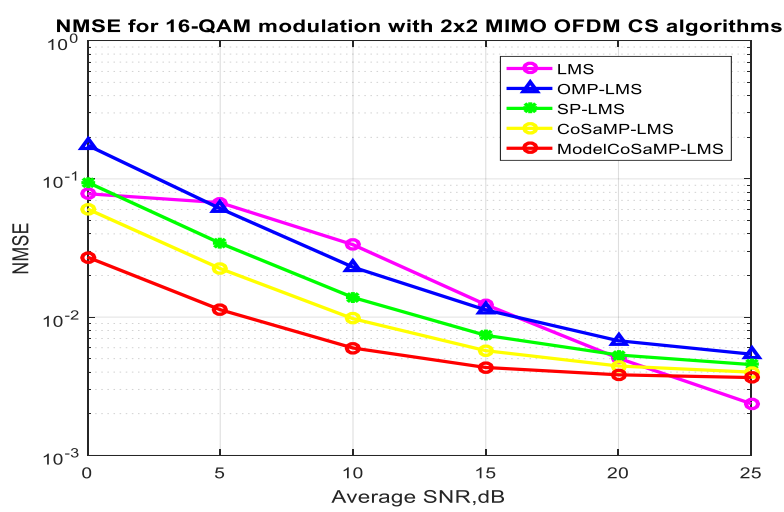

Fig. 5: NMSE Vs SNR (Db) For Conventional CS with Model-Based Cosamp for MIMO-OFDM Channel Estimation with LMS Tool.
In Fig.5 comparison of performance of conventional and the model-based compression technique with LMS tool for MIMOOFDM system channel estimation are shown. LMS tool uses the adaptive technique for channel estimation. It can be calculated from the results that NMSE reduces by $34 \%$ using model-based CoSaMP combined with LMS tool for MIMO OFDM channel estimation.

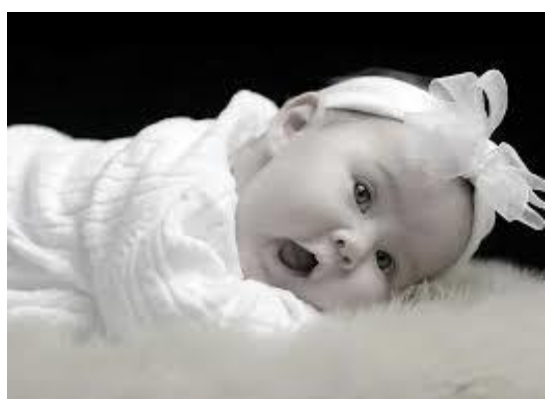

Fig. 6: Original Image Considered with 1024x768pixcels.

Figure 6 shows the original image considered for the MIMO OFDM channel estimation using conventional CoSaMP and the model based CoSaMP with LMS tool.
(A)

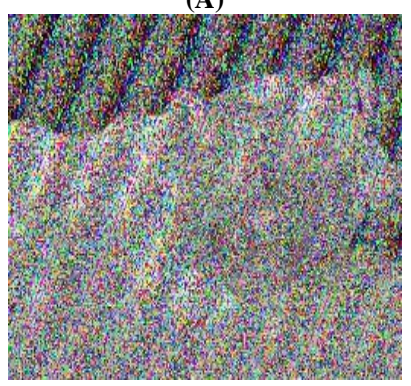

(B)

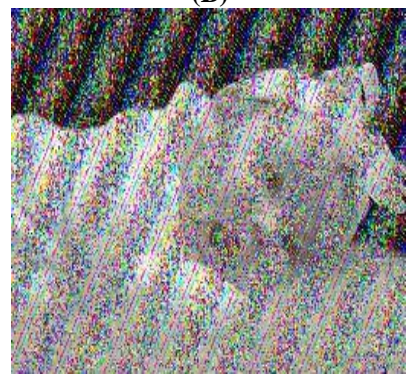

Fig. 7: (a) Reconstructed output image at $S N R=5$ for CosaMP (b) Reconstructed output image at SNR $=5$ for Model based CosaMP.
(C)

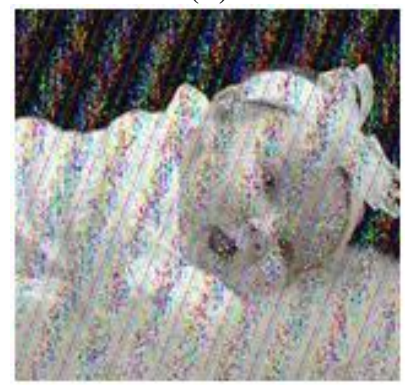

(D)

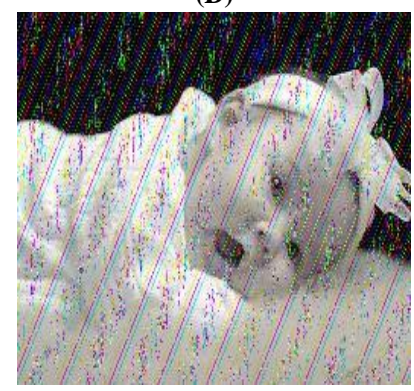

Fig. 7: (C) Reconstructed Output Image at SNR $=10$ for Cosamp (D) Reconstructed Output Image at SNR $=10$ for Model Based Cosamp.
(E)

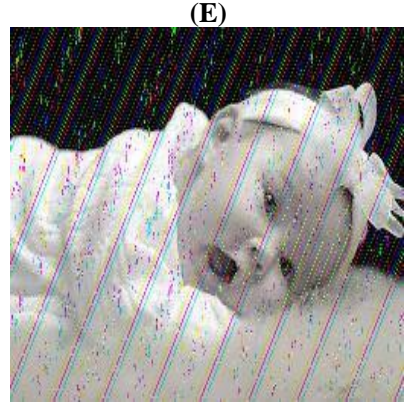

(F)

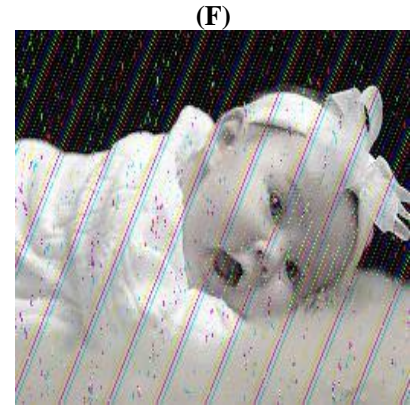

Fig. 7: (E) Reconstructed Output Image at SNR $=15$ for Cosamp (F) Reconstructed Output Image at SNR $=15$ for Model Based Cosamp.

Fig.7 shows the reconstructed images for CoSaMP and the model based CoSaMP for the SNR values 5, 10 and 15 dBs.The Mean Square Error(MSE) is calculated for the conventional CoSaMp and the model based CoSaMP for the at different SNR values for 
the reconstructed images. MSE is less in the model based CoSaMP thanconventional CoSaMP,hence the reconstruction is better. These results are as tabulated in Table 3 for $\mathrm{SNR}=5,10$ and $15 \mathrm{~dB}$. In addition, the Fig. 8 shows the graphical representation of these results.Peak signal to noise ratio (PSNR) is measure to find the quality of reconstrucgion. The quality of the reconstruction is superios if the the PSNR is high.For $r$ the model based CoSaMP is comparatively more than conventional CoSaMP as tabulated in table 4 . Figure 9 shows these results graphically.

Table 3: Minimum Square Error at Different SNR Values for Cosamp and Model-Based Cosamp

\begin{tabular}{llll}
\hline & $\begin{array}{l}\text { MSE at } \\
\text { SNR }=5 \mathrm{~dB}\end{array}$ & $\begin{array}{l}\text { MSE at } \\
\text { SNR=10dB }\end{array}$ & $\begin{array}{l}\text { MSE at } \\
\text { SNR=15dB }\end{array}$ \\
\hline $\begin{array}{l}\text { CoSaMP } \\
\text { Modelbased }\end{array}$ & 5310 & 1420 & 463 \\
CoSaMP & 3030 & 670 & 270 \\
\hline
\end{tabular}

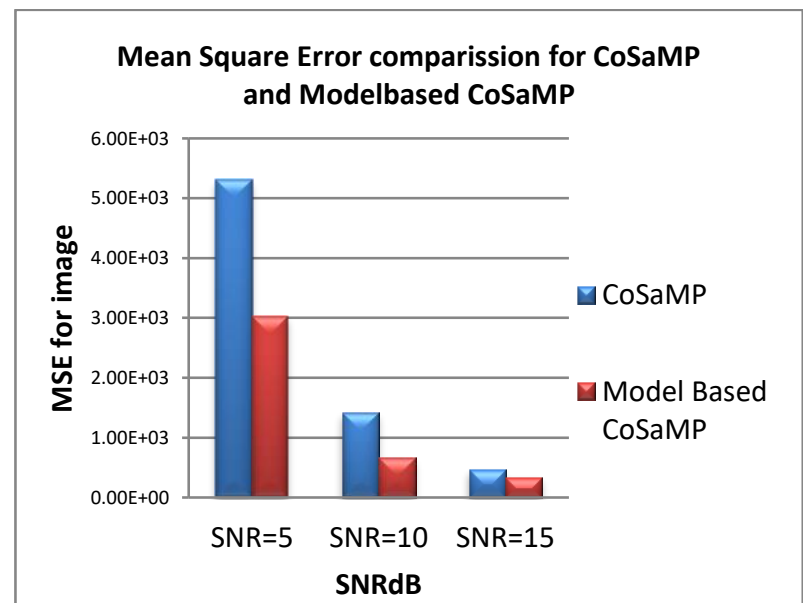

Fig. 8: Mean Square Error Comparison for Cosamp and Model Based Cosamp

Table 4: Peak to Signal Noise Ratio at Different SNR Values for Cosamp and Model-Based Cosamp

\begin{tabular}{llll}
\hline & PSNR at & PSNR at & PSNR at \\
& SNR=5dB & SNR=10dB & SNR=15dB \\
\hline $\begin{array}{l}\text { CoSaMP } \\
\text { Model based }\end{array}$ & $10.8816 \mathrm{~dB}$ & $16.618 \mathrm{~dB}$ & $20.4742 \mathrm{~dB}$ \\
CoSaMp & $13.3125 \mathrm{~dB}$ & $19.8761 \mathrm{~dB}$ & $23.6625 \mathrm{~dB}$ \\
\hline
\end{tabular}

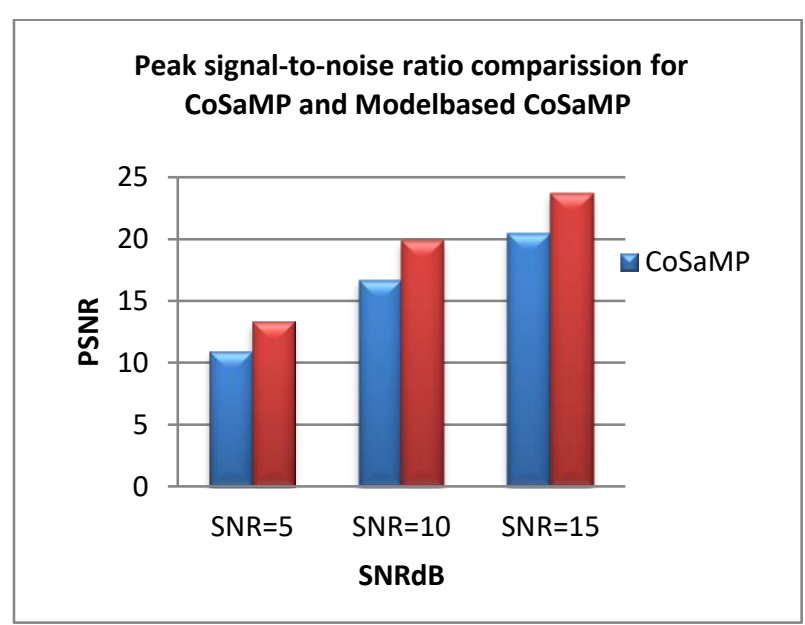

Fig. 9: PSNR Comparison of Cosamp and Model Based Cosamp.

\section{Conclusion}

MIMO OFDM channel is estimated using conventional and model-basedCoSaMPwithLS and LMS tools. Simulated results shows among conventional CS algorithms CoSaMP with LMS tool has the best performance than other conventional CS algorithms considered. And MIMO OFDM channel estimation using model- based CoSaMP with LMS gives the $34 \%$ more performance. The MSE and PSNR is calculated for the conventional and the model based CoSaMP at $\mathrm{SNR}=5,10$ and $15 \mathrm{~dB}$. MSE is reduced $57 \%, 47$ $\%$ and $40 \%$ at $\mathrm{SNR}=5,10$ and $15 \mathrm{~dB}$ respectively for model-based CoSaMP and PSNR increases by $30 \%$ at $\mathrm{SNR}=5,10$ and $15 \mathrm{~dB}$. The computational complexity is also calculated and it is less for model based CoSaMP as tabulated in the table 1 . The work can be extended using model-based Iterative Soft thresholding (IST) for highly multipath channels for MIMO-OFDM systems.

\section{References}

[1] Li, Y., "Simplified Channel Estimation for OFDM Systems with Multiple Transmit Antennas," IEEE Transactions on Communications, vol. 1, pp. 67-75, January 2002.

[2] W. U. Bajwa, J. Haupt, A. M. Sayeed and R. Nowak, "Compressed channel sensing: a new approach toestimating sparse multipath channels," Proc. IEEE, vol. 98,no. 6, pp. 1058-1076, June 2010 https://doi.org/10.1109/JPROC.2010.2042415.

[3] Z. H. Wang, C. R. Berger, J. Z. Huang and S. L. Zhou, "Application for Compressive Sensing to Sparse Channel Estimation", IEEE Communication Magazine, vol. 48, pp.164-174, November 2010. https://doi.org/10.1109/MCOM.2010.5621984.

[4] R. F. Song, X. Y. He, , and K. Q. Zhou, "Study ofCompressive Sensing Based Sparse Channel Estimation in OFDM Systems" Journal of Nanjing University of PostsandTelecommunications(Natural Science), vol. 30, no. 2,pp. 60-65, 2011.

[5] Jung Nam Ba et.al, "Performance of Multi-user MIMO OFDM Channel Estimation with LS and MMSE for 802.11n Systems", IEEE Int. conf communication and Information Technology, pp. 15, .Sept.2009.

[6] X. Yang, Y. X. Peng, X. F. Zhang, W. B. Wang and B. Wu, "Compressed MIMO-OFDM Channel Estimation,"Communication Technology IEEE InternationalConferenceon, pp.1291-1294, November 2010.

[7] P N Jayanthi, andS. Ravishankar,"Sparse channel estimation for MIMO-OFDM systems using compressed sensing" IEEE International Conference on Recent Trends in Electronics, Information \& Communication Technology (RTEICT), pp: 1060-1064., 2016.

[8] J. A. Tropp and D. Needell, "Cosamp:iterative signal recovery from incomplete and inaccurate measurements," Appl. Comput. Harm. Anal., pp. 301-321, 2009.

[9] Richard G. Baraniuk, Volkan Cevher; Marco F. Duarte; Chinmay Hegde,"Model-Based Compressive Sensing“.,IEEE Transactions on Information Theory Volume: 56, Issue: 4 pp: 1982 - 2001, 2010

[10] D. L. Donoho, "Compressed sensing," IEEE Trans. Inf. Theory, vol.52, no. 4, pp. 1289-1306, Sep. 2006. https://doi.org/10.1109/TIT.2006.871582.

[11] E. J. Candès, "Compressive Sampling," in Proc. Int. Congr. Math.,Madrid, Spain, 2006, vol. 3, pp. 1433-1452.

[12] R. G. Baraniuk, "Compressive sensing” IEEE Signal Processing Mag.,vol. 24, no. 4, pp. 118-120, Jul. 2007, 124.

[13] E.J Jose et. al. "Signal recovery for random projections," in Proc. Compute. Imaging III at SPIE Electron. Imaging vol. 5674, pp. 7686. Jan. 2005.

[14] Qi and L. Wu, "A hybrid compressed sensing algorithm for sparse channel estimation forMIMO OFDM systems," in Proc. IEEE Int. Conf.Acoust., Speech, Signal Process., May 2011, pp. 3488-3491.

[15] J. A. Tropp and A. C. Gilbert, "Signal Recovery From Random Measurements via OMP," IEEE Trans. Inf. Theory, vol. 53, no. 12, pp. 4655- 4666, Dec. 2007. https://doi.org/10.1109/TIT.2007.909108.

[16] W. Dai and O. Milenkovic, "Subspace Pursuit for Compressive Sensing Signal Reconstruction," IEEE Trans. Inf. Theory, vol. 55, no. 5, pp. 2230-2249, Apr. 2009. https://doi.org/10.1109/TIT.2009.2016006. 